\title{
Reference To Study Results Uniform Resource Locator
}

National Cancer Institute

\section{Source}

National Cancer Institute. Reference To Study Results Uniform Resource Locator. NCI

Thesaurus. Code C94046.

A complete reference to a website (including http://) that is directly relevant to the study. 